\title{
Role of GOLPH3 and GOLPH3L in the proliferation of human rhabdomyosarcoma
}

\author{
OSAMU KUNIGOU ${ }^{1}$, HIROKO NAGAO ${ }^{1}$, NAOYA KAWABATA ${ }^{1}$, YASUHIRO ISHIDOU ${ }^{2}$, \\ SATOSHI NAGANO $^{1}$, SHINGO MAEDA ${ }^{2}$, SETSURO KOMIYA ${ }^{1}$ and TAKAO SETOGUCHI ${ }^{3}$ \\ ${ }^{1}$ Department of Orthopaedic Surgery, ${ }^{2}$ Department of Medical Joint Materials, ${ }^{3}$ The Near-Future Locomotor \\ Organ Medicine Creation Course (Kusunoki Kai), Graduate School of Medical and \\ Dental Sciences, Kagoshima University, Kagoshima 890-8520, Japan
}

Received June 14, 2011; Accepted July 18, 2011

DOI: 10.3892/or.2011.1413

\begin{abstract}
GOLPH3 was originally identified by proteomic analyses of Golgi proteins localized in the trans-Golgi network. Recently, it was reported that GOLPH3 is up-regulated in various types of malignancies, including melanoma, colon cancer and lung cancer. However, the mechanism through which GOLPH3 is involved in the pathogenesis of rhabdomyosarcoma remains unidentified. In order to explore the function of GOLPH3 and its isoform, GOLPH3L, in the pathogenesis of rhabdomyosarcoma, we investigated the expression and knockdown effects of GOLPH3 and GOLPH3L in human rhabdomyosarcoma. Western blot analysis and real-time PCR revealed that human rhabdomyosarcoma cell lines and biopsy specimens exhibited an increased expression of GOLPH3 and GOLPH3L. GOLPH3 and GOLPH3L knockdown by siRNA prevented the proliferation of human rhabdomyosarcoma cell lines. In addition, double-knockdown of GOLPH3 and GOLPH3L also prevented the proliferation of rhabdomyosarcoma cell lines. Our findings improve the understanding of rhabdomyosarcoma pathogenesis and suggest that the knockdown of GOLPH3 or GOLPH3L may be an effective treatment for rhabdomyosarcoma.
\end{abstract}

\section{Introduction}

Rhabdomyosarcoma, which is the most common soft-tissue sarcoma in children, includes two major subtypes, alveolar rhabdomyosarcoma and embryonal rhabdomyosarcoma. Approximately $70 \%$ of rhabdomyosarcoma patients without metastatic tumors are cured, but the prognosis of patients with metastasis or relapse remains dismal. Although numerous clinical trials have been performed, little improvement has

Correspondence to: Dr Takao Setoguchi, The Near-Future Locomotor Organ Medicine Creation Course (Kusunoki Kai), Graduate School of Medical and Dental Sciences, Kagoshima University, 8-35-1 Sakuragaoka, Kagoshima 890-8520, Japan

E-mail: setoro@m2.kufm.kagoshima-u.ac.jp

Key words: rhabdomyosarcoma, GOLPH3, GOLPH3L, cell proliferation, Golgi apparatus been made for high-risk rhabdomyosarcoma patients, who have a 3 -year overall survival of approximately $30 \%(1,2)$. Thus, improvements in outcome and in the understanding of the molecular pathways of rhabdomyosarcoma pathogenesis are a priority.

GOLPH3 (also known as GOPP1, GPP34, MIDAS or FLJ90675) was originally identified by proteomic analyses of Golgi proteins localized to the trans-Golgi network $(3,4)$. It has been reported that GOLPH3 is dynamically associated with the trans-Golgi matrix, as it rapidly moves from the transGolgi network to the cytosol and localizes in endosomes and at the plasma membrane (5). Recently, it has been reported that GOLPH3 modulates solid tumors through mTOR signaling (6).

GOLPH3L (also known as GPP34R, GMx33 $\beta$ or FLJ10687) is an isoform of GOLPH3; these proteins are very homologous to each other and are similarly localized (4). Although the two isoforms are highly homologous in their amino acid sequences, the function of GOLPH3L has not yet been examined.

In the present study, we investigated the involvement of GOLPH3 and GOLPH3L in the pathogenesis of human rhabdomyosarcoma. We assessed the expression of GOLPH3 and GOLPH3L in rhabdomyosarcoma and the effect of GOLPH3 or GOLPH3L inhibition on the growth of rhabdomyosarcoma cells. We found that the knockdown of GOLPH3 or GOLPH3L inhibited human rhabdomyosarcoma cell proliferation.

\section{Materials and methods}

Cell culture. The human rhabdomyosarcoma cell lines, KYM-1 and RD, were provided by the Health Science Research Resources Bank (HSRRB, Osaka, Japan). The human rhabdomyosarcoma cell line A204 was provided by the American Type Culture Collection (Manassas, VA, USA). HSKMC normal myoblast cells were purchased from Toyobo (Osaka, Japan). Cells were cultured in Dulbecco's modified Eagle's medium (DMEM) supplemented with 10\% FBS, penicillin (100 U/ml), and streptomycin $(100 \mu \mathrm{g} / \mathrm{ml})$. In all experiments, cells were maintained in 100-mm culture dishes (Nunc, New York, NY, USA) at $37^{\circ} \mathrm{C}$ in a humidified $5 \% \mathrm{CO}_{2} / 95 \%$ air atmosphere.

Patient specimens. We obtained 2 biopsy specimens of human rhabdomyosarcoma from primary lesions. Pathological 


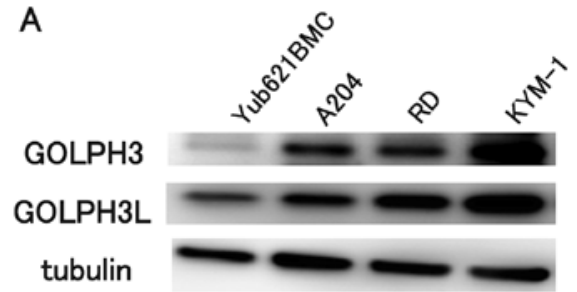

B

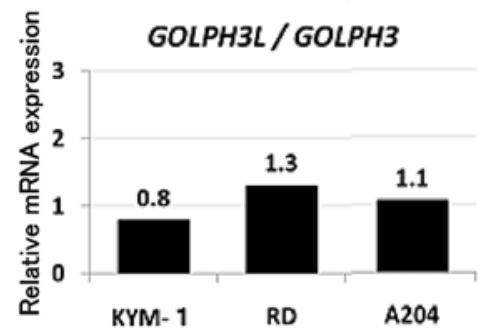

Figure 1. Up-regulation of GOLPH3 and GOLPH3L in human rhabdomyosarcoma cell lines. The protein extracted from 3 rhabdomyosarcoma cell lines and 1 mesenchymal stem cell line (Yub621BMC) was used for Western blot analysis. Western blot analysis results showed that the 3 human rhabdomyosarcoma cell lines exhibited increased GOLPH3 and GOLPH3L expression compared to the mesenchymal stem cells (A). The total RNA extracted from the 3 rhabdomyosarcoma cell lines was used for real-time PCR. The real-time PCR results revealed that the 3 human rhabdomyosarcoma cell lines expressed GOLPH3L from 0.8- to 1.3-fold the level of expression of GOLPH3 (B).

examination revealed that patient 1 had embryonal rhabdomyosarcoma and patient 2 had alveolar rhabdomyosarcoma. Biopsy was performed before chemotherapy or radiotherapy for diagnosis. Control muscle was obtained from surgery for scoliosis. The study protocol was approved by the Institutional Review Board of Kagoshima University. All patients and controls provided written informed consent.

Real-time PCR. Real-time PCR was performed as previously reported (7). Briefly, each primer set used amplified a 160 to 200 bp amplicon. Reactions were run using SYBR Green (BioRad, Hercules, CA, USA) on a MiniOpticon ${ }^{\mathrm{TM}}$ machine (Bio-Rad). The comparative $\mathrm{Ct}(\Delta \Delta \mathrm{Ct})$ method was used to evaluate the fold change of mRNA expression. All PCR reactions were performed in triplicate, with 3 different concentrations of cDNA. All primers were designed using Primer3 software (http://frodo.wi.mit.edu/cgi-bin/primer3/primer3.cgi). The comparative $\mathrm{Ct}(\Delta \Delta \mathrm{Ct})$ analysis method was used to evaluate the fold change of mRNA expression, using expression of $G A P D H$ as a reference. The following primers were used: GOLPH3, 5'-AAGCCGTTCTTGACAAATGG-3' and 5'-GGT GTTGGCCTTCAGACATT-3'; GOLPH3L, 5'-ATGACCCTC AGCGTATGGAC-3' and 5'-TTTGTCCCTTCCACTTC AGG-3'; GAPDH, 5'-GAAGGTGAAGGTCGGAGTC-3' and 5'-GAAGATGGTGATGGGATTTC-3'.

Western blot analysis. Western blot analysis was performed as previously reported (8). Approximately $20 \mu \mathrm{g}$ of protein was loaded onto $4-12 \%$ NuPAGE precast gels (Invitrogen Corp., Carlsbad, CA, USA) and transferred to nitrocellulose membranes. The membranes were blocked in TBST buffer
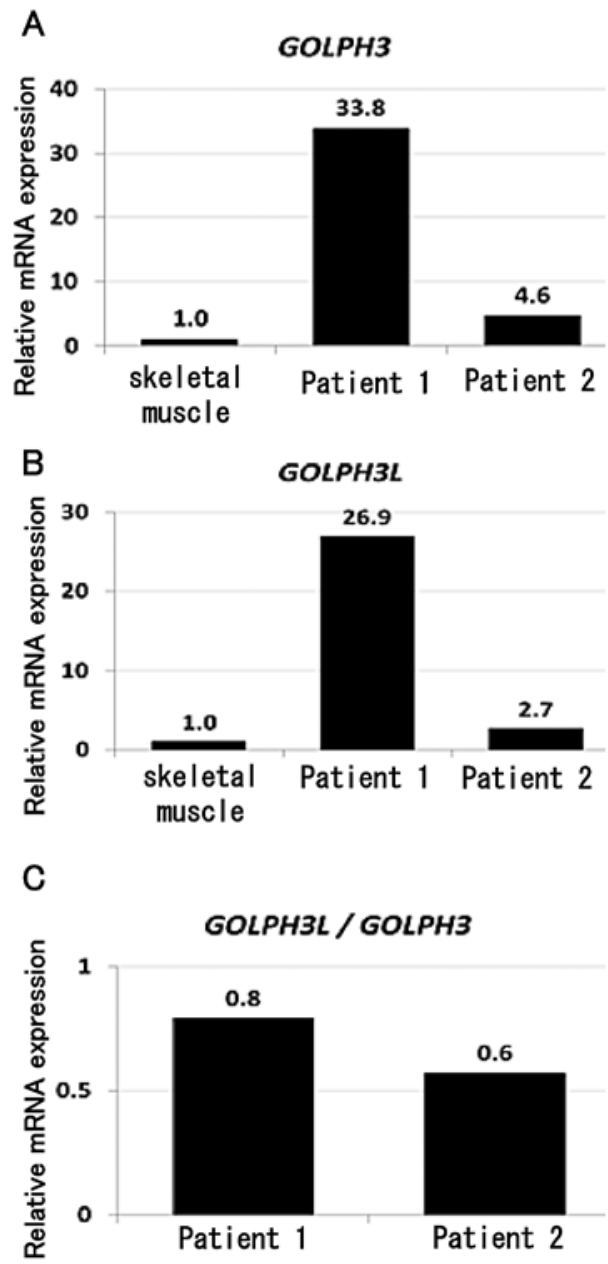

Figure 2. Up-regulation of GOLPH3 and GOLPH3L in human rhabdomyosarcoma biopsy specimens. Real-time PCR was performed to analyze the gene expression in 2 biopsy samples obtained from 2 patients. The results indicated that the expression of GOLPH3 was increased from 4.6- to 33.8fold (A) and that of GOLPH3L was increased from 2.7- to 26.9-fold (B) in the 2 samples. The real-time PCR results showed that the 2 human rhabdomyosarcoma biopsy specimens expressed GOLPH3L from 0.6- to 0.8-fold the level of expression of $G O L P H 3(\mathrm{C})$. The experiment was repeated in triplicate with similar results.

containing $10 \%$ milk for $1 \mathrm{~h}$ and then incubated with antibodies diluted in TBST. The following antibodies were used: anti-GOLPH3, anti-GOLPH3L (Sigma-Aldrich Co., St. Louis, MO, USA) and anti-tubulin (Santa Cruz Biotechnology, Inc., Santa Cruz, CA, USA). Detection was performed using the ECL detection system (GE Healthcare Life Sciences, Giles, UK). Protein bands were visualized by LAS-4000mini (GE Healthcare Life Sciences, Tokyo, Japan).

MTT assay. The 3-(4,5-dimethylthiazol-2-yl)-2,5-diphenyltetrazolium bromide (MTT) assay was used to evaluate cell proliferation, as previously described (9). Briefly, cells cultured on microplates were incubated with the MTT substrate for $4 \mathrm{~h}$, rinsed with PBS, and subsequently lysed. The developed optical density was analyzed with a microplate reader (Bio-Rad Laboratories). GOLPH3 siRNA and GOLPH3L siRNA were purchased from Santa Cruz Biotechnology. Transfection of the siRNAs was performed according to the supplier's recommendations using Lipofectamine RNAiMax (Invitrogen). 

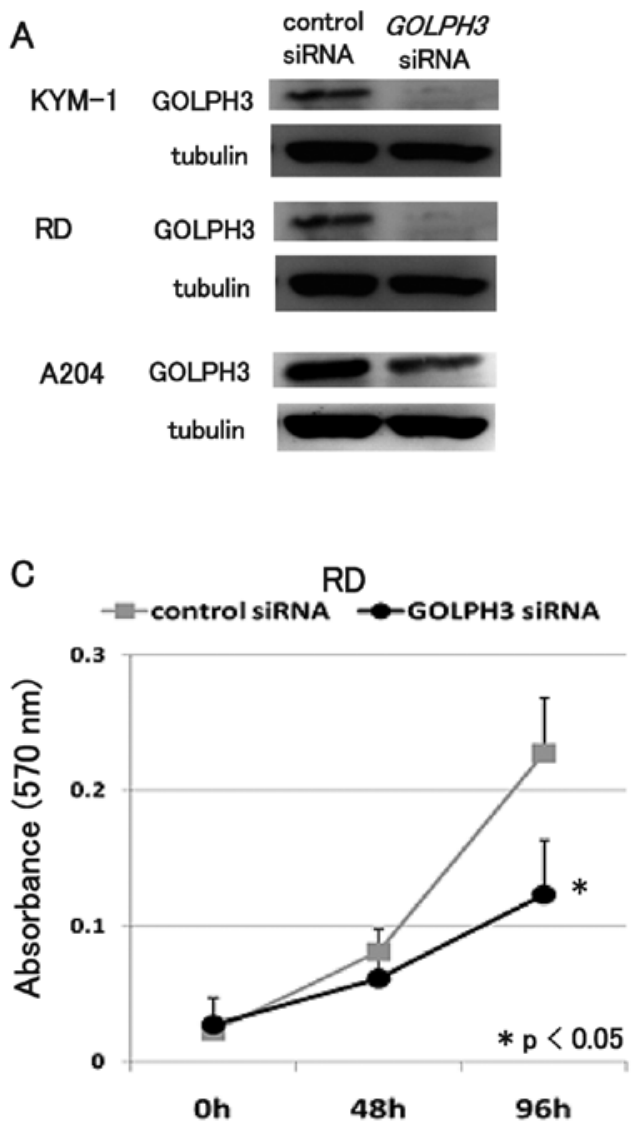

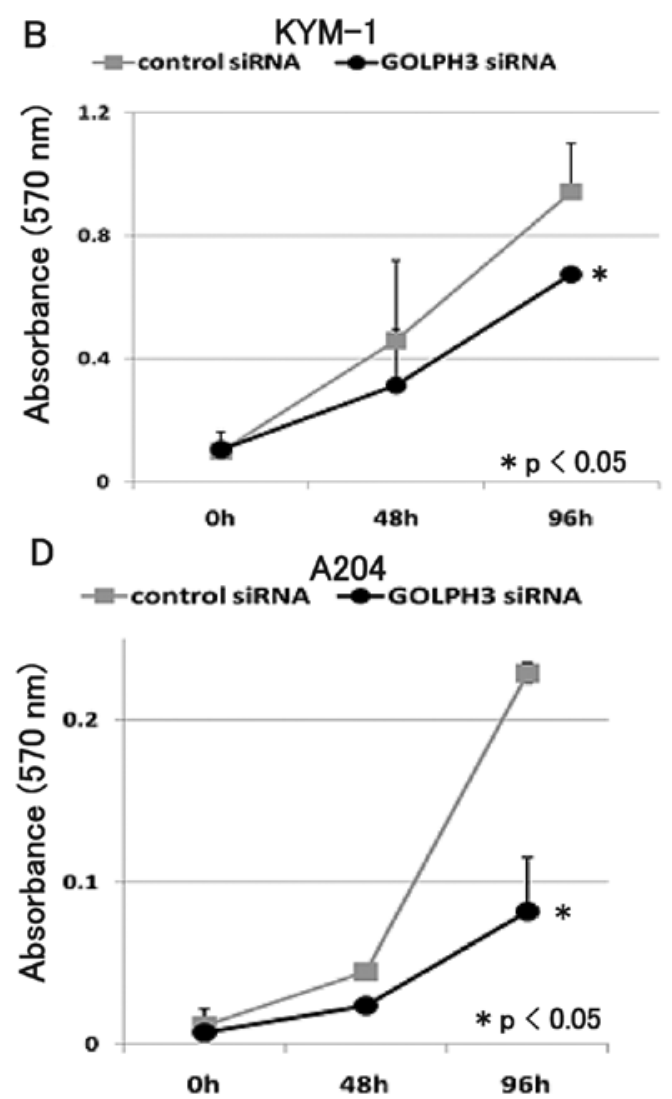

Figure 3. Knockdown of GOLPH3 inhibits human rhabdomyosarcoma cell proliferation. Western blot analysis revealed that lysates of GOLPH3 siRNAtransfected cells exhibited decreased GOLPH3 expression (A). MTT assay revealed that knockdown of GOLPH3 resulted in a decrease in the growth of viable KYM-1 (B), RD (C) and A204 (D) cells over $96 \mathrm{~h}\left({ }^{*} \mathrm{P}<0.05\right)$. These experiments were performed in triplicate with similar results (error bars represent SD).

Statistical analysis. All experiments were performed 3 times, except where otherwise stated, and samples were analyzed in triplicate. For real-time PCR experiments, each sample was tested using 3 different cDNA concentrations. Results are presented as mean (SD). The statistical differences between groups were assessed by applying a Student's t-test for unpaired data, using Microsoft Office Excel (Microsoft Corp., Redmond, WA, USA) and Statistica (StatSoft Inc., Tulsa, OK, USA).

\section{Results}

Overexpression of GOLPH3 and GOLPH3L in human rhabdomyosarcoma cell lines. In order to assess the role of GOLPH3, we examined the expression of GOLPH3 in human rhabdomyosarcoma cell lines. Western blot analysis showed that the expression of GOLPH3 was up-regulated in human rhabdomyosarcoma cell lines compared to mesenchymal stem cells. Furthermore, Western blot analysis showed that the expression of GOLPH3L, which is an isoform of GOLPH3, was also up-regulated in human rhabdomyosarcoma cell lines (Fig. 1A). It has been reported that GOLPH3L is expressed at levels of $<2 \%$ in HeLa cells (10). Our Western blot results showed that both GOLPH3 and GOLPH3L resulted in intense bands. In order to compare the expression of GOLPH3 and GOLPH3L, we performed real-time PCR. Real-time PCR showed that the expression of $G O L P H 3 L$ was 0.8 - to 1.3 -fold the expression of GOLPH3 (Fig. 1B).

Overexpression of GOLPH3 and GOLPH3L in human rhabdomyosarcoma biopsy specimens. We examined the expression of GOLPH3 and GOLPH3L with real-time PCR. Real-time PCR revealed that the expression of GOLPH3 and $G O L P H 3 L$ was up-regulated between 4.6- and 33.8-fold and between 2.7- and 26.9-fold, respectively, in the 2 biopsy samples compared to normal skeletal muscle (Fig. 2A and B). Furthermore, real-time PCR revealed that the expression of GOLPH3L was 0.6- to 0.8 -fold the expression of $G O L P H 3$ (Fig. 2C). These findings suggest that the expression of GOLPH3 and GOLPH3L is up-regulated in human rhabdomyosarcoma.

Knockdown of GOLPH3 and GOLPH3L prevents human rhabdomyosarcoma cell proliferation. In order to examine the function of GOLPH3 in human rhabdomyosarcoma, the expression of GOLPH3 was knocked down with siRNA. Scrambled siRNA was used as a negative control. Western blot analysis showed that GOLPH3 siRNA decreased the expression of GOLPH3 in KYM-1, RD, and A204 human rhabdomyosarcoma cell lines (Fig. 3A). MTT assay showed that knockdown of GOLPH3 inhibited the proliferation of KYM-1, RD and A204 cells (Fig. 3B-D). We next examined 
A

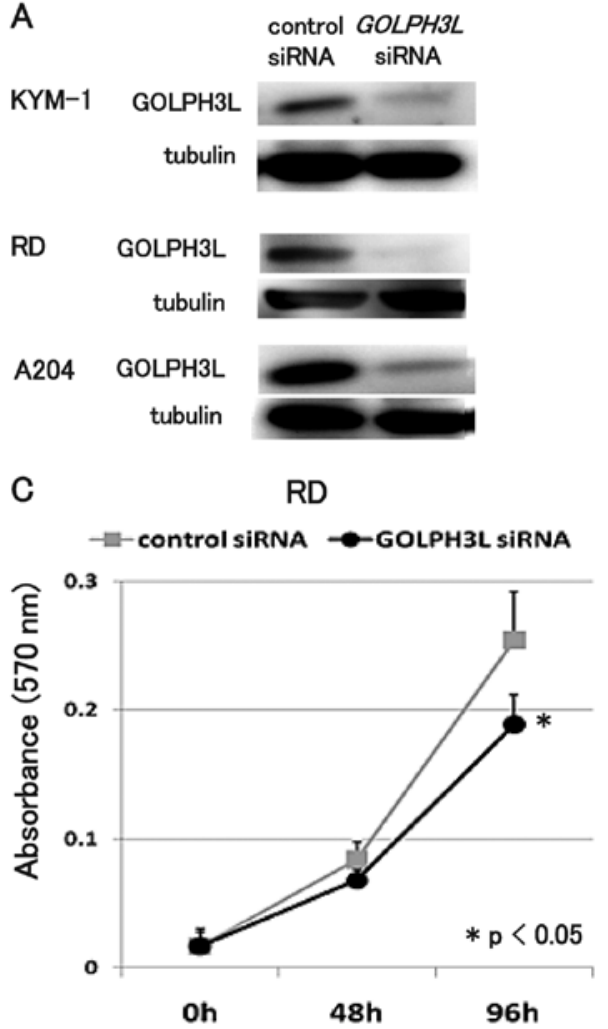

B

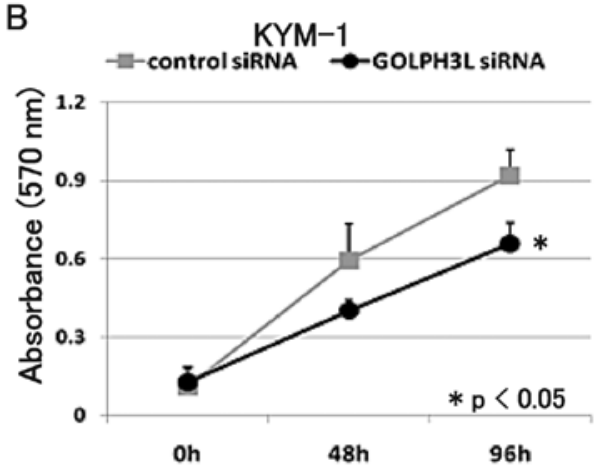

D

A204

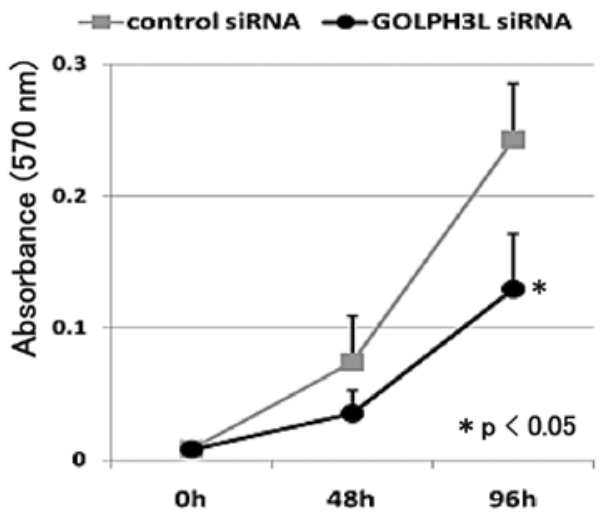

Figure 4. Knockdown of GOLPH3L inhibits human rhabdomyosarcoma cell proliferation. Western blot analysis revealed that lysates of GOLPH3L siRNAtransfected cells decreased GOLPH3L expression (A). The MTT assay revealed that the knockdown of GOLPH3L resulted in a decrease in the growth of viable KYM-1 (B), RD (C) and A204 (D) cells over 96 h ( $\left.{ }^{*}<<0.05\right)$. These experiments were performed in triplicate with similar results (error bars represent SD).

the effects of GOLPH3L knockdown on rhabdomyosarcoma growth. Western blot analysis showed that GOLPH3L siRNA decreased the expression of GOLPH3L in the KYM-1, RD, and A204 cells (Fig. 4A). MTT assay showed that knockdown of GOLPH3L inhibited the proliferation of KYM-1, RD and A204 cells (Fig. 4B-D). Furthermore, we assessed the effect of knockdown of both GOLPH3 and GOLPH3L together. MTT assay showed that knockdown of GOLPH3 and GOLPH3L inhibited the proliferation of KYM-1, RD, and A204 cells (Fig. 5). These findings revealed that GOLPH3 and GOLPH3L knockdown inhibits human rhabdomyosarcoma growth.

\section{Discussion}

GMx33 is conserved from yeast to mammals, in which two very homologous forms, $\alpha$ and $\beta$ have been noted (4). The human orthologs of GMx33 $\alpha$ and $\beta$ (GOLPH3 and GOLPH3L, respectively) were simultaneously identified through a separate proteomic analysis and described as Golgi-localized proteins $(3,4)$. In addition, GOLPH3 has a large cytoplasmic pool (3). Cell-based assays confirmed that GOLPH3 can exchange between cytosolic and Golgi-associated pools rapidly, and the protein was additionally found to be associated with tubules and vesicles leaving the Golgi apparatus (5). In addition, GOLPH3 is localized to mitochondria (10). GOLPH3 is up-regulated in response to mitochondrial dysfunction in the muscle of mitochondrial disease (10). These findings suggest that GOLPH3 has various cytochemical functions other than the functions associated with being a Golgi-related protein.

We showed that the knockdown of GOLPH3 prevents the proliferation of human rhabdomyosarcoma. Although the precise function of GOLPH3 as an oncogene has not been elucidated, GOLPH3 has been implicated in protein trafficking, receptor recycling, and glycosylation, which are all processes involved in tumorigenesis $(6,11)$. In addition, GOLPH3 interacts with a retromer complex, which is responsible for protein trafficking between endosomes and the trans-Golgi network $(6,12)$. A role of the Golgi network in cancer pathogenesis has been reported, in that some oncoproteins, including RAS, are functionally active when localized in the Golgi network $(13,14)$. GOLPH3 interacts with the VPS35 subunit of the retromer protein-recycling complex and enhances signaling through the mammalian target of rapamycin, which integrates input from multiple signaling pathways to control cell growth, proliferation, and survival (15). In addition, GOLPH3 enhances downstream growth signaling in response to receptor tyrosine kinase activation (6). The function of these molecular pathways in rhabdomyosarcoma pathogenesis should be examined.

An analysis of copy number alterations identified frequent regional amplification at 5p13 in multiple tumor types, including breast, colorectal and lung cancer (6). They reported that $5 \mathrm{p} 13$ copy number status was significantly correlated with gene expression of GOLPH3 in human lung cancer specimens, and subsequent functional studies pointed to GOLPH3 as a target for activation in cancers with 5 p gain $(6,11)$. We 
A
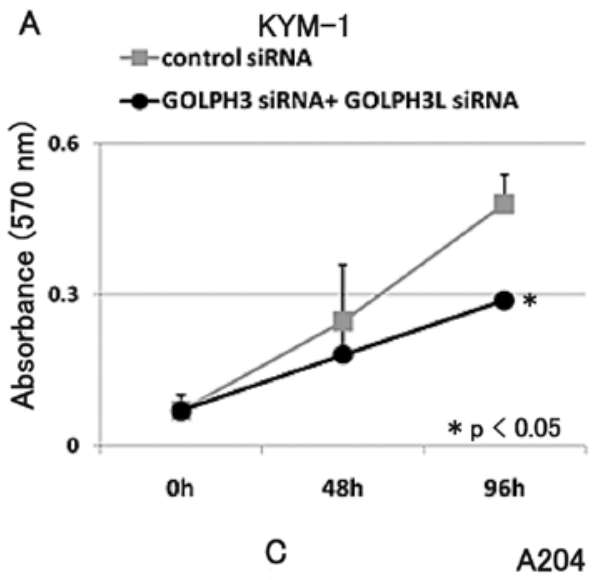

- - control siRNA

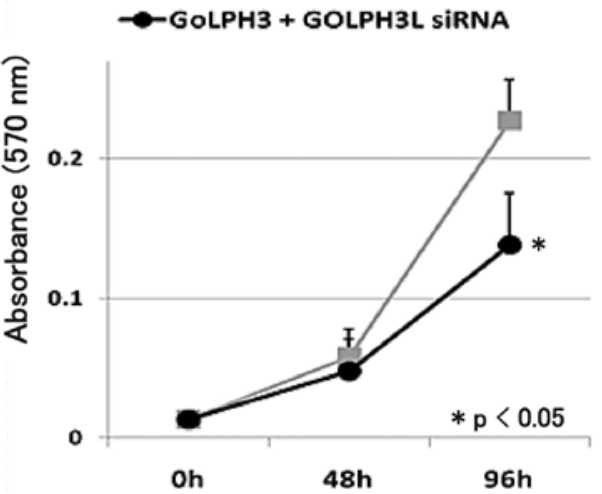

B - RD

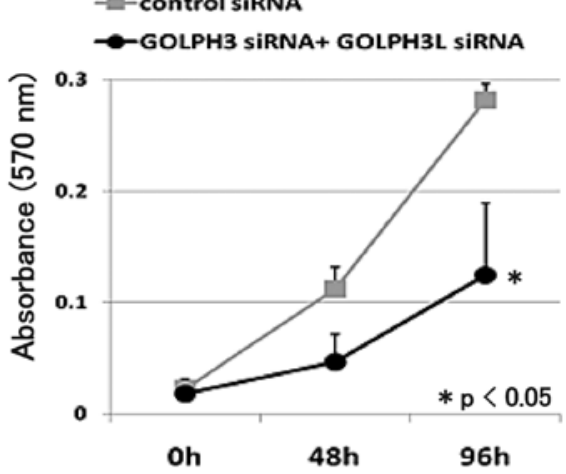

5nockdown of GOLPH3 and GOLPH3L inhibits human rhabdomyosarcoma cell proliferation. The MTT assay revealed that the knockdown of GOLPH3 and GOLPH3L resulted in a decrease in the growth of viable KYM-1 (A), RD (B), and A204 (C) cells over $96 \mathrm{~h}$. These experiments were performed in triplicate with similar results (error bars represent $\mathrm{SD} ;{ }^{*} \mathrm{P}<0.05$ ).

showed that the expression of GOLPH3 and GOLPH3L is up-regulated in human rhabdomyosarcoma cell lines and biopsy specimens. GOLPH3L is located at 1q21.3. It has been reported that 1q21 is amplified in several human malignancies, including rhabdomyosarcoma (16-24). An amplification of 5 p13 has not yet been reported in rhabdomyosarcoma. Another mechanism including epigenetic modification may affect the up-regulation of GOLPH3 and GOLPH3L as the process of gene transcription is regulated through the orchestration of many transcription factors and epigenetic mechanisms $(25,26)$.

It has been reported that GOLPH3 is ubiquitously expressed and is not cell-specific (3). These findings suggest that knockdown of GOLPH3 results in toxicity in normal GOLPH3-expressing cells. Scott et al reported that the knockdown of GOLPH3 in low GOLPH3-expressing cells showed a minimal effect on cell proliferation, indicating that acute GOLPH3 depletion is not generally toxic to all cells (6). These finding suggest that the inhibition of GOLPH3 is an effective treatment for malignant tumors with GOLPH3 up-regulation.

GOLPH3L was also identified as a Golgi-localized protein with a large cytosolic pool $(3,4)$. GOLPH3L was found to be expressed at a very decreased level compared to GOLPH3 in HeLa and NRK cells $(5,10)$. Our Western blot analysis showed that GOLPH3L was expressed in a significant amount in bone marrow mesenchymal stem cells and human rhabdomyosarcoma stem cells, and GOLPH3L-specific siRNA reduced that expression. In addition, real-time PCR showed that
GOLPH3L expression was abundant compared to GOLPH3 in RD, KYM-1 and A204 cells. These findings suggest that both GOLPH3 and GOLPH3L are expressed in human rhabdomyosarcoma cells. Although GOLPH3 and GOLPH3L share significant homology, there are different regions that have different functions. To the best of our knowledge, the function of GOLPH3L has not yet been examined. We showed that the knockdown of GOLPH3L prevents the proliferation of human rhabdomyosarcoma cell lines. These findings revealed that not only GOLPH3, but also GOLPH3L, may have oncogenic functions and that inhibition of GOLPH3L may be an effective treatment for malignant tumors with GOLPH3L up-regulation.

We performed an MTT assay following the double-knockdown of GOLPH3 and GOLPH3L. Although the MTT assay showed that double-knockdown prevents rhabdomyosarcoma cell proliferation statistically, double-knockdown did not have a synergistic effect. Real-time PCR showed that the knockdown efficiency of GOLPH3 or GOLPH3L siRNA was between 61 and $84 \%$ (data not shown). These findings suggest that the low levels of expression of GOLPH3 or GOLPH3L following siRNA treatment are due to an effect of cell proliferation. In addition, it has been reported that the deletion of yeast GOLPH3 (VPS74) had no effect on viability and growth (27). These findings suggest that the knockdown of GOLPH3 and GOLPH3L treatment does not inhibit tumor cell proliferation completely. To examine the precise function of GOLPH3 and GOLPH3L, a deletion examination in mammalian cells is needed. 
In conclusion, our findings demonstrated that the expression of GOLPH3 and GOLPH3L was up-regulated in human rhabdomyosarcoma. These findings improve our understanding of rhabdomyosarcoma pathogenesis and suggest that the knockdown of GOLPH3 or GOLPH3Lis an effective treatment for rhabdomyosarcoma.

\section{Acknowledgements}

We are grateful to Hui Gao for the excellent technical assistance. This study was supported by Grants-in-Aid for Scientific Research (KAKENHI) (B) 18390419, (C) 19591725, (C) 20591786, (C) 21591919, (C) 21591920, and (C) 22591663 , and a Grant-in-Aid from the Ministry of Health, Labour, and Welfare of Japan for the Third Term Comprehensive Control Research for Cancer.

\section{References}

1. Oberlin O, Rey A, Lyden E, et al: Prognostic factors in metastatic rhabdomyosarcomas: results of a pooled analysis from United States and European cooperative groups. J Clin Oncol 26: 2384-2389, 2008

2. Huh WW and Skapek SX: Childhood rhabdomyosarcoma: new insight on biology and treatment. Curr Oncol Rep 12: 402-410, 2010 .

3. Wu CC, Taylor RS, Lane DR, Ladinsky MS, Weisz JA and Howell KE: GMx33: a novel family of trans-Golgi proteins identified by proteomics. Traffic 1: 963-975, 2000.

4. Bell AW, Ward MA, Blackstock WP, et al: Proteomics characterization of abundant Golgi membrane proteins. J Biol Chem 276: $5152-5165,2001$

5. Snyder CM, Mardones GA, Ladinsky MS and Howell KE: GMx33 associates with the trans-Golgi matrix in a dynamic manner and sorts within tubules exiting the Golgi. Mol Biol Cell 17: 511-524, 2006.

6. Scott KL, Kabbarah O, Liang MC, et al: GOLPH3 modulates mTOR signalling and rapamycin sensitivity in cancer. Nature 459: 1085-1090, 2009 .

7. Hirotsu M, Setoguchi T, Matsunoshita Y, et al: Tumour formation by single fibroblast growth factor receptor 3-positive rhabdomyosarcoma-initiating cells. Br J Cancer 101: 2030-2037, 2009.

8. Hirotsu M, Setoguchi T, Sasaki H, et al: Smoothened as a new therapeutic target for human osteosarcoma. Mol Cancer 9: 5, 2010.

9. Matsunoshita Y, Ijiri K, Ishidou Y, et al: Suppression of osteosarcoma cell invasion by chemotherapy is mediated by urokinase plasminogen activator activity via up-regulation of EGR1. PLoS One 6: e16234, 2011.
10. Nakashima-Kamimura N, Asoh S, Ishibashi Y, et al: MIDAS/ GPP34, a nuclear gene product, regulates total mitochondrial mass in response to mitochondrial dysfunction. J Cell Sci 118: 5357-5367, 2005.

11. Scott KL and Chin L: Signaling from the Golgi: mechanisms and models for Golgi phosphoprotein 3-mediated oncogenesis. Clin Cancer Res 16: 2229-2234, 2010.

12. Bonifacino JS and Hurley JH: Retromer. Curr Opin Cell Biol 20: 427-436, 2008

13. Chiu VK, Bivona T, Hach A, et al: Ras signalling on the endoplasmic reticulum and the Golgi. Nat Cell Biol 4: 343-350, 2002.

14. Eaton S: Retromer retrieves wntless. Dev Cell 14: 4-6, 2008.

15. Wullschleger S, Loewith $\mathrm{R}$ and Hall MN: TOR signaling in growth and metabolism. Cell 124: 471-484, 2006.

16. Knuutila S, Aalto Y, Autio K, et al: DNA copy number losses in human neoplasms. Am J Pathol 155: 683-694, 1999.

17. Larramendy ML, Lushnikova T, Bjorkqvist AM, et al: Comparative genomic hybridization reveals complex genetic changes in primary breast cancer tumors and their cell lines. Cancer Genet Cytogenet 119: 132-138, 2000.

18. Guan XY, Fang Y, Sham JS, et al: Recurrent chromosome alterations in hepatocellular carcinoma detected by comparative genomic hybridization. Genes Chromosomes Cancer 29: 110-116, 2000.

19. Mairal A, Pinglier E, Gilbert E, et al: Detection of chromosome imbalances in retinoblastoma by parallel karyotype and CGH analyses. Genes Chromosomes Cancer 28: 370-379, 2000.

20. Szymanska J, Virolainen M, Tarkkanen M, et al: Overrepresentation of $1 \mathrm{q} 21-23$ and $12 \mathrm{q} 13-21$ in lipoma-like liposarcomas but not in benign lipomas: a comparative genomic hybridization study. Cancer Genet Cytogenet 99: 14-18, 1997.

21. Tarkkanen M, Karhu R, Kallioniemi A, et al: Gains and losses of DNA sequences in osteosarcomas by comparative genomic hybridization. Cancer Res 55: 1334-1338, 1995.

22. Meza-Zepeda LA, Forus A, Lygren B, et al: Positional cloning identifies a novel cyclophilin as a candidate amplified oncogene in 1q21. Oncogene 21: 2261-2269, 2002.

23. Atiye J, Wolf M, Kaur S, et al: Gene amplifications in osteosarcoma-CGH microarray analysis. Genes Chromosomes Cancer 42: 158-163, 2005.

24. Sadikovic B, Yoshimoto M, Chilton-MacNeill S, Thorner P, Squire JA and Zielenska M: Identification of interactive networks of gene expression associated with osteosarcoma oncogenesis by integrated molecular profiling. Hum Mol Genet 18: 1962-1975, 2009.

25. Jaenisch R and Bird A: Epigenetic regulation of gene expression: how the genome integrates intrinsic and environmental signals. Nat Genet 33 (Suppl): 245-254, 2003.

26. Grewal SI and Moazed D: Heterochromatin and epigenetic control of gene expression. Science 301: 798-802, 2003.

27. Winzeler EA, Shoemaker DD, Astromoff A, et al: Functional characterization of the $S$. cerevisiae genome by gene deletion and parallel analysis. Science 285: 901-906, 1999. 